\title{
Características clínicas, sociodemográficas e desfechos de gestantes hospitalizadas com COVID-19
}

Clinical, sociodemographic and outcome characteristics of pregnant women hospitalized with COVID-19

Características clínicas, sociodemográficas y de evolución de las embarazadas hospitalizadas por COVID-19

Jaqueline Guimarães Elói de Brito ORCID: https://orcid.org/0000-0002-5200-6506 Universidade Federal de Sergipe, Brasil E-mail: jaquebriito@gmail.com

Cleyse Caroline Alves de Alencar ORCID: https://orcid.org/0000-0001-8120-7668 Universidade Federal de Sergipe, Brasil E-mail: cleysealencar@gmail.com Amanda Camilo Lemos ORCID: https://orcid.org/0000-0002-1796-6448 Universidade Federal de Sergipe, Brasil

E-mail: amandacamilolemos@gmail.com

Caroline Lima dos Reis Caetano ORCID: https://orcid.org/0000-0003-2661-7116 Universidade Federal de Sergipe, Brasil E-mail: carolinelimareis@hotmail.com Max Oliveira Menezes ORCID: https://orcid.org/0000-0001-7660-4458 Universidade Tiradentes, Brasil E-mail: maxoliver19@hotmail.com

Maria do Socorro Claudino Barreiro ORCID: https://orcid.org/0000-0001-9823-4638 Universidade Federal de Sergipe, Brasil

E-mail: socorroclaudino@academico.ufs.br

\section{Resumo}

O presente estudo tem por objetivo descrever as características clínicas e epidemiológicas, fatores de risco e desfecho da COVID-19 em gestantes. Trata-se de um estudo quantitativo com abordagem descritiva. A amostra foi composta por 54 voluntárias assistidas em uma maternidade de alto risco localizada em Aracaju, Sergipe, Brasil. Os dados foram extraídos de prontuários e foram analisadas as variáveis sociodemográficas maternas relativas à assistência prénatal, ao parto e ao puerpério imediato, a presença ou não de sintomas de COVID-19, internação em UTI e desfecho. Os dados foram descritos por meio de frequências absolutas e relativa. Foi também calculada a média, desvio padrão, valor mínimo e valor máximo das variáveis quantitativas no IBM® SPSS. Predominaram gestantes com faixa etária de 20 a 34 anos $(64,8 \% ; n=35)$ e pardas $(61,1 \% ; n=33)$. Com relação à via de parto, destacaram-se as cesáreas $(76,9 \% ; n=30)$. Houve destaque para sintomas como cefaléia $(51,8 \% ; n=28)$, febre $(51,8 \% ; n=28)$, tosse $(44,4 \%$; $\mathrm{n}=24)$, coriza $(35,1 \% ; \mathrm{n}=19)$ e dispnéia $(20,3 \% ; \mathrm{n}=11)$. A maioria das participantes apresentou, pelo menos, algum sinal de gravidade $(87 \% ; n=47)$ e possuíam comorbidades $(77,7 \% ; n=42)$. Foram registrados três óbitos maternos. Conclui-se que a presença de comorbidade foi um fator de risco relevante nas participantes que desenvolveram doença mais grave, indicando possivelmente um perfil pré-definido que contribui para um maior risco de resultados maternos graves.

Palavras-chave: Gravidez; Coronavírus; Infecções por Coronavírus; Mortalidade materna; Complicações na gravidez.

\section{Abstract}

This study aims to describe the clinical and epidemiological characteristics, risk factors and outcome of COVID-19 in pregnant women. This is a quantitative study with a descriptive approach. The sample consisted of 54 volunteers assisted in a high-risk maternity hospital located in Aracaju, Sergipe, Brazil. Data were extracted from medical records and analyzed as maternal sociodemographic variables related to prenatal care, participation and immediate 
puerperium, the presence or absence of symptoms of COVID-19, admission to the ICU and outcome. Data were generated using absolute and related frequencies. It was also calculated to mean, standard deviation, minimum value, and maximum value of the quantitative variables in IBM® SPSS. Pregnant women aged between 20 and 34 years $(64.8 \% ; n=35)$ and brown $(61.1 \% ; n=33)$ predominated. Regarding the mode of delivery, cesarean sections stood out $(76.9 \% ; n=30)$. Symptoms such as headache $(51.8 \% ; n=28)$, fever $(51.8 \% ; n=28)$, cough (44.4\%; n=24), runny nose $(35.1 \% ; n=19)$ and dyspnea $(20.3 \% ; n=11)$. Most participants had at least some sign of severity $(87 \% ; n=47)$ and had comorbidities $(77.7 \% ; \mathrm{n}=42)$. Three maternal deaths were registered. It is concluded that the presence of comorbidity was a relevant risk factor in participants who developed more severe disease, possibly indicating a predefined profile that contributes to a higher risk of severe maternal outcomes.

Keywords: Pregnancy; Coronavirus; Coronavirus infections; Maternal mortality; Pregnancy complications.

\section{Resumen}

Este estudio tiene como objetivo describir las características clínicas, epidemiológicas, factores de riesgo y evolución del COVID-19 en mujeres embarazadas. Se trata de un estudio cuantitativo con enfoque descriptivo. La muestra estuvo constituida por 54 voluntarias atendidas en una maternidad de alto riesgo ubicada en Aracaju, Sergipe, Brasil. Se extrajeron los datos de las historias clínicas y se analizaron las variables sociodemográficas maternas relacionadas con la atención prenatal, el parto y el puerperio inmediato, la presencia o ausencia de síntomas de COVID-19, el ingreso a la UCI y el resultado. Los datos se describieron utilizando frecuencias absolutas y relativas. Los media, desviación estándar, valor mínimo y valor máximo de variables cuantitativas en IBM® SPSS. Predominaron las gestantes entre 20 y 34 años $(64,8 \% ; n=35)$ y morenas $(61,1 \% ; n=33)$. En cuanto a la modalidad de parto, destacaron las cesáreas $(76,9 \% ; n=30)$. Síntomas como cefalea $(51,8 \% ; n=28)$, fiebre $(51,8 \% ; n=28)$, tos $(44,4 \% ; n$ $=24)$, secreción nasal $(35,1 \% ; n=19)$ y disnea $(20,3 \% ; n=11)$. La mayoría de los participantes tenían al menos algún signo de gravedad $(87 \% ; n=47)$ y tenían comorbilidades $(77,7 \% ; n=42)$. Se registraron tres muertes maternas. Se concluye que la presencia de comorbilidad fue un factor de riesgo relevante en las participantes que desarrollaron una enfermedad más grave, posiblemente indicando un perfil predefinido que contribuye a un mayor riesgo de resultados maternos graves.

Palabras clave: Embarazo; Coronavirus; Infecciones por coronavirus; Mortalidad materna; Complicaciones del embarazo.

\section{Introdução}

Em março de 2020, a Organização Mundial da Saúde (2020) declarou uma pandemia devido à propagação global e à calamidade alcançada pela infecção do SARS-CoV-2. No entanto, nesse contexto de pandemia, o conhecimento sobre o impacto dessa infecção era, ainda, limitado e persistem dúvidas em relação aos riscos à gestação associada à COVID-19, visto que, o período gestacional é considerado pelo Ministério da Saúde (2020) como grupo de risco e propenso a desenvolver sintomas graves da COVID-19. Esse risco aumentado pode estar relacionado à imunodeficiência relativa da gravidez associada a adaptações do organismo materno na gestação, parto e pós-parto (Schwartz \& Dhaliwal, 2020). Também se destacam as alterações anatômicas e fisiológicas, cardiorrespiratórias e o aumento da coagulabilidade sanguínea, podendo piorar o prognóstico das doenças (Dashraath et al., 2020).

De acordo com o Ministério da Saúde (2021), no Brasil, o atendimento de pré-natal de risco habitual de pacientes sem COVID-19 ou com suspeita ou confirmação de COVID-19 com sintomas leves deve ser realizado na Atenção Primária. Já para as maternidades de alto risco, são encaminhadas as gestantes e parturientes de alto risco sem COVID-19 e com suspeita ou confirmação de COVID-19 com indicação de internação. O modelo assistencial adotado em Sergipe com relação ao fluxo de regulação das gestantes seguiu o preconizado pelo Ministério da Saúde e, apenas as gestantes e parturientes com complicações maternas foram encaminhadas para maternidade de alto risco, independente da suspeita ou confirmação de COVID-19 (Brasil, 2021).

De modo geral, as gestantes brasileiras são predominantemente jovens e hígidas, o que pode propiciar formas leves da doença, conforme demonstraram estudos iniciais (Yu et al., 2020. Wen et al., 2020). Contudo, recentes publicações evidenciaram formas graves e relatos de morte materna, sobretudo em mulheres imunocomprometidas e com comorbidades como hipertensão, diabetes, asma e cardiopatias (Knight et al., 2020. Souza \& Amorim, 2021. Takemoto et al., 2020).

Nesse contexto e levando-se em consideração que a pandemia de COVID-19 tornou-se um problema de saúde pública 
dentro do contexto brasileiro, devem ser realizados estudos durante o ciclo gravídico-puerperal, para produzir resultados que orientem decisões políticas para a melhoria da qualidade do cuidado à saúde materna nos serviços de atendimento à saúde, visando um melhor prognóstico da doença, garantir assistência adequada e prevenir desfechos desfavoráveis a saúde maternoinfantil (Allotey et al., 2020).

Dessa maneira, justifica-se a relevância do presente estudo ao contribuir significativamente para o conhecimento das variáveis associadas à COVID-19, a partir da descrição das características sociodemográficas maternas (idade, renda, escolaridade e zona de moradia), do tipo de serviço, hipótese diagnóstica, comorbidade e categoria profissional que realiza a assistência. Como também, se a gestante apresentou sintomatologia para a SARS-CoV-2 e se o desfecho foi favorável, evoluindo para cura com a alta da paciente, ou com a ocorrência de complicação materna, com necessidade de transferência e internação em Unidade de Terapia Intensiva (UTI) e/ou óbito.

Diante disso, objetivou-se descrever as características clínicas e epidemiológicas, fatores de risco e desfecho da COVID-19 em gestantes.

\section{Metodologia}

Trata-se de um estudo quantitativo com abordagem descritiva, realizado entre maio e dezembro de 2020. Foram avaliadas 54 gestantes e puérperas, através da análise de prontuário materno e do recém-nascido e da ficha de notificação para Síndrome Respiratória Aguda Grave. Essa ficha alimenta o Sistema de Informação de Vigilância Epidemiológica da Gripe SIVEP-Gripe, gerido pelo Ministério da Saúde, em conjunto com as Secretarias Estaduais e Municipais de Saúde.

A pesquisa foi realizada em uma maternidade pública que é referência estadual para o atendimento de gestantes de alto risco obstétrico, localizada em Aracaju, Sergipe, Brasil.

Essa maternidade dispõe de 7 leitos para observação na admissão, 33 leitos para tratamento clínico de gestantes, 33 leitos para alojamento conjunto (binômio mãe e bebê), 16 leitos cirúrgicos (entre pré-parto, sala de recuperação e salas cirúrgicas) para realização de procedimentos obstétricos e cirurgias pediátricas, 34 leitos de Unidade de Terapia Intensiva Neonatal (UTIN), 25 leitos de Unidade de Cuidado Intermediário Neonatal Convencional (UCINco), 27 leitos de Unidade de Cuidado Intermediário Neonatal Canguru (UCINca). No entanto, a instituição não possui leitos de UTI materna, sendo necessário regular a paciente para os hospitais que possuem leitos de Unidades de Terapia Intensiva (UTI) vinculados ao SUS.

Diante do cenário de pandemia, foi elaborado um plano de contingência com abertura de uma Unidade de Cuidados Especiais que dispõe de 1 leito para gestante/puérpera e 6 leitos para o recém-nascido e foram criados 6 leitos de isolamento para internamento clínico das gestantes.

As pacientes foram selecionadas a partir de uma listagem de internação diária, sendo consideradas elegíveis todas as mulheres que residem no Estado de Sergipe e que, durante a gestação, parto ou puerpério imediato, apresentaram infecção confirmada pelo SARS-CoV-2, através da lista de pacientes positivas da Comissão de Controle de Infecção Hospitalar e do Laboratório da maternidade, por meio de Reação em cadeia da Polimerase em tempo real - RT-PCR ou teste rápido (imunocromatográfico). Foram excluídas da pesquisa as mulheres readmitidas após 10 dias de alta hospitalar e com prontuários com escrita inelegível.

O questionário utilizado no presente estudo foi adaptado da Pesquisa Nascer no Brasil (Fiocruz, 2020) e do Protocolo de Atendimento de Pacientes com COVID-19 do Instituto de Saúde Elpídio de Almeida - ISEA (Amorim, Melo, Neto \& Oliveira, 2020), sendo compostos por questões objetivas relacionadas às condições sociodemográficas, à assistência pré-natal, ao parto e ao puerpério imediato, diagnóstico, comorbidades, desfecho, presença ou não de sintomas e realização de teste para COVID-19.

A análise estatística foi realizada no IBM® SPSS 20.0 Mac (IBM® SPSS - StatisticalPackage for the Social Sciences 
20.0 Mac (SPSS 20.0 Mac, SPSS Inc., Chicago, Illinois, EUA). Foi calculada a média, desvio padrão, valor mínimo e valor máximo das variáveis quantitativas. Os dados foram descritos por meio de frequências absolutas e relativa/proporcional.

Este estudo foi autorizado pela instituição na qual a pesquisa foi realizada, mediante a assinatura da carta de anuência e do termo de autorização de uso de arquivos/dados de pesquisa, e foi aprovado pelo Comitê de Ética em Pesquisa da Universidade Federal de Sergipe, sob Parecer no 4889554.

Todos os cuidados foram adotados visando garantir o sigilo e confidencialidade das informações, por meio da assinatura do termo de compromisso e confidencialidade pelos pesquisadores. As puérperas que aceitaram participar deste estudo assinaram o Termo de Compromisso Livre e Esclarecido, com garantia de recusa a qualquer momento, sem o sofrimento de quaisquer danos por parte da instituição.

Os pesquisadores seguiram as diretrizes e normas regulamentadoras preconizadas na Resolução $n^{\circ} 466 / 12$ do Conselho Nacional de Saúde sobre as pesquisas envolvendo seres humanos (Brasil, 2012).

\section{Resultados}

A média e desvio-padrão de idade das puérperas diagnosticadas com a COVID-19 durante a gravidez foi de 27,93 $\pm 7,46$ anos, sendo 15 a mínima e 44 a máxima. A maioria encontrava-se na faixa etária de 20 a 34 anos $(64,81 \%$; n= 35), cor da pele parda $(61,11 \% ; n=33)$, residia em zona urbana $(85,19 \% ; n=46)$, cursou ensino fundamental ou médio $(66,66 \%$; $=$ 36) e possuía trabalho remunerado $(57,41 \% ; n=31)$ (Tabela 1$)$. 
Tabela 1 - Resultados descritivos do perfil sociodemográfico das puérperas diagnosticadas com a COVID-19 durante a gravidez. Aracaju, Sergipe, Brasil, 2020.

\begin{tabular}{|c|c|c|}
\hline Perfil sociodemográfico e de saúde & $\mathrm{N}=54$ & $\%$ \\
\hline \multicolumn{3}{|l|}{ Faixa etária } \\
\hline$\leq 19$ anos & 07 & 12,96 \\
\hline 20 a 34 anos & 35 & 64,81 \\
\hline$\notin 35$ anos & 12 & 22,22 \\
\hline \multicolumn{3}{|l|}{ Cor da pele } \\
\hline Preta & 08 & 14,81 \\
\hline Parda & 33 & 61,11 \\
\hline Branca & 05 & 9,26 \\
\hline Ignorada & 08 & 14,81 \\
\hline \multicolumn{3}{|l|}{ Zona de moradia } \\
\hline Urbana & 46 & 85,19 \\
\hline Rural & 08 & 14,81 \\
\hline \multicolumn{3}{|l|}{ Escolaridade } \\
\hline Analfabeto & 01 & 1,85 \\
\hline Fundamental & 14 & 25,93 \\
\hline Médio & 22 & 40,74 \\
\hline Superior & 04 & 7,41 \\
\hline Ignorado & 13 & 24,07 \\
\hline \multicolumn{3}{|l|}{ Situação conjugal } \\
\hline Vive com companheiro(a) & 06 & 11,11 \\
\hline Vive sem companheiro(a) & 26 & 48,15 \\
\hline Ignorado & 22 & 40,74 \\
\hline \multicolumn{3}{|l|}{ Ocupação } \\
\hline Possui trabalho remunerado & 31 & 57,41 \\
\hline Não possui trabalho remunerado & 18 & 33,33 \\
\hline Ignorado & 05 & 9,26 \\
\hline \multicolumn{3}{|l|}{\begin{tabular}{|l|} 
Possui comorbidades \\
\end{tabular}} \\
\hline Sim & 42 & 77,78 \\
\hline Não & 12 & 22,22 \\
\hline \multicolumn{3}{|l|}{ Faz uso de bebida alcoólica } \\
\hline Sim & 00 & 0,00 \\
\hline Não & 29 & 53,70 \\
\hline Ignorado & 25 & 46,30 \\
\hline \multicolumn{3}{|l|}{ Faz uso de cigarro } \\
\hline Sim & 03 & 5,56 \\
\hline Não & 27 & 50,00 \\
\hline Ignorado & 24 & 44,44 \\
\hline
\end{tabular}

Nota: N= Frequência absoluta, \%= Frequência relativa. Fonte: Serviço de Arquivo Médico - SAME (2020).

A maioria das mulheres avaliadas não eram primigestas $(75,93 \% ; n=41)$, não era primípara $(68,52 \%$; $n=37)$, estavam no terceiro trimestre da gravidez $(81,4 \% ; n=44)$, realizou o pré-natal da gravidez atual $(74,07 \%$; n= 40) e teve gestação única/não gemelar $(92,59 \% ; n=50)$. Ademais, observa-se predomínio de realização de partos por via cirúrgica/cesariana 
(76,92\%; $n=30)$ e condução da parturição pela categoria médica $(94,87 \% ; n=37)$ (Tabela 2). É importante ressaltar que 15 pacientes foram internadas apenas para tratamento clínico, sem necessidade de interromper a gravidez.

Tabela 2 - Resultados descritivos do perfil ginecológico e obstétrico das puérperas diagnosticadas com a COVID-19 durante a gravidez. Aracaju, Sergipe, Brasil, 2020.

\begin{tabular}{|c|c|c|}
\hline Perfil ginecológico e obstétrico & $\mathbf{N}=\mathbf{5 4}$ & $\%$ \\
\hline \multicolumn{3}{|l|}{ Número de gestações (incluindo a gravidez atual) } \\
\hline $1^{\text {a } \text { gestação (primigesta) }}$ & 13 & 24,07 \\
\hline $2^{\text {a gestação }}$ & 14 & 25,93 \\
\hline 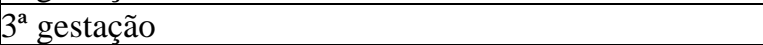 & 10 & 18,52 \\
\hline $4^{\mathrm{a}}$ gestação & 04 & 7,41 \\
\hline $5^{\text {a }}$ ou maior número de gestações & 13 & 24,07 \\
\hline \multicolumn{3}{|l|}{\begin{tabular}{|l} 
Número de partos (incluindo a gravidez atual) \\
\end{tabular}} \\
\hline $1^{\circ}$ parto (primípara) & 17 & 31,48 \\
\hline $2^{\circ}$ parto & 06 & 11,11 \\
\hline $3^{\circ}$ parto & 03 & 5,56 \\
\hline $4^{\circ}$ parto & 02 & 3,70 \\
\hline $5^{\circ}$ ou maior número de partos & 26 & 48,15 \\
\hline \multicolumn{3}{|l|}{ Realizou pré-natal na gravidez atual } \\
\hline Sim & 40 & 74,07 \\
\hline Não & 00 & 0,00 \\
\hline Ignorado & 14 & 25,93 \\
\hline \multicolumn{3}{|l|}{ Tipo de gestação (gravidez atual) } \\
\hline Abortamento & 01 & 1,85 \\
\hline Gemelar & 03 & 5,56 \\
\hline Única & 50 & 92,59 \\
\hline \multicolumn{3}{|l|}{ Tipo de parto realizado (gravidez atual) } \\
\hline Cesariana & 30 & 76,92 \\
\hline Vaginal & 09 & 23,08 \\
\hline \multicolumn{3}{|c|}{ Categoria profissional que conduziu o parto (gravidez atual) } \\
\hline Enfermeiro & 01 & 2,56 \\
\hline Médico & 37 & 94,87 \\
\hline Parto em trânsito & 01 & 2,56 \\
\hline \multicolumn{3}{|l|}{ Hipótese diagnóstica } \\
\hline Pré-Eclâmpsia grave & 09 & 16,67 \\
\hline Pré-Eclâmpsia Leve & 05 & 9,26 \\
\hline Oligodrâmnio & 04 & 7,41 \\
\hline Restrição de Crescimento Intra-Uterino & 07 & 12,96 \\
\hline Diabetes Mellitus Gestacional & 11 & 20,37 \\
\hline Depressão & 02 & 3,70 \\
\hline Infecção do Trato Urinário & 07 & 12,96 \\
\hline Abortamento incompleto & 01 & 1,85 \\
\hline Pielonefrite & 04 & 7,41 \\
\hline Anemia & 02 & 3,70 \\
\hline Hipertensão Arterial Crônica & 08 & 14,81 \\
\hline Hipertensão Gestacional & 05 & 9,26 \\
\hline Síndrome HELLP & 04 & 7,41 \\
\hline Edema Agudo de Pulmão & 01 & 1,85 \\
\hline
\end{tabular}




\begin{tabular}{|c|c|c|}
\hline Ameaça de Trabalho de Parto Prematuro & 03 & 5,56 \\
\hline Trabalho de Parto Prematuro & 08 & 14,81 \\
\hline Ruptura Prematura de Membranas & 07 & 12,96 \\
\hline Diabetes Mellitus Tipo II & 02 & 3,70 \\
\hline Diabetes Mellitus Tipo I & 01 & 1,85 \\
\hline Anidrâmnio & 01 & 1,85 \\
\hline Sífilis & 03 & 5,56 \\
\hline Febre Reumática & 01 & 1,85 \\
\hline Obesidade & 02 & 3,70 \\
\hline Taquicardia Fetal & 02 & 3,70 \\
\hline Asma & 05 & 9,26 \\
\hline Cardiopatia & 03 & 5,56 \\
\hline Eclâmpsia & 04 & 7,41 \\
\hline Pré-Eclâmpsia sobreposta & 03 & 5,56 \\
\hline Descolamento Prematuro de Placenta & 02 & 3,70 \\
\hline Bradicardia Fetal & 03 & 5,56 \\
\hline Encefalopatia & 01 & 1,85 \\
\hline Vasculite de Sistema Nervoso Central & 01 & 1,85 \\
\hline Plaquetopenia & 02 & 3,70 \\
\hline Corioamnionite & 01 & 1,85 \\
\hline Hepatite B & 01 & 1,85 \\
\hline
\end{tabular}

Nota: N= Frequência absoluta, \%= Frequência relativa. Fonte: Serviço de Arquivo Médico - SAME (2020).

Verificou-se que $42(77,7 \%)$ pacientes apresentaram comorbidades. Entre estas, as mais frequentes foram: Hipertensão Crônica (25,9\%; n= 14), Diabetes Mellitus Gestacional (20,3\%; n= 11), Hipertensão Gestacional (9,2\%; n= 5) e Asma $(9,2 \% ; n=5)$.

No que diz respeito à hipótese diagnóstica, as patologias mais frequentes foram o Diabetes Mellitus Gestacional (20,37\%; n= 11), Pré-Eclâmpsia Grave (16,67\%; n= 9), Hipertensão Crônica (HAC) (14,81\%; n= 8), Trabalho de Parto Prematuro $(14,81 \%$; $n=8)$, Restrição de Crescimento Intra-Uterino (RCIU) $(12,96 \%$; n= 7), Ruptura Prematura de Membrana $(12,96 \% ; n=7)$, Pré-Eclâmpsia (PE) Leve $(9,26 \% ; n=5)$ e Eclâmpsia $(7,41 \% ; n=4)$.

Os principais sinais/sintomas obstétricos que motivaram a consulta no serviço especializado foram dor em Baixo Ventre (DBV) $(38,89 \% ; n=21)$, cefaleia $(37,04 \% ; n=20)$ e Pressão Arterial (PA) elevada $(31,48 \%$; n=17). Em relação à sintomatologia da COVID-19, houve predomínio de relatos maternos de cefaleia $(51,85 \%$; $n=28)$, febre $(51,85 \%$; $n=28)$, tosse $(44,44 \% ; n=24)$ e coriza $(35,19 \% ; n=19)$. Ressalta-se que mais da metade das mulheres avaliadas apresentou aumento de Proteína C Reativa - PCR (55,56\%; n=30). Outros sinais/sintomas de gravidade da COVID-19 prevalentes foram leucocitose $(37,04 \% ; n=20)$, aumento da frequência cardíaca (FC) $(27,78 \% ; n=15)$ e ausculta pulmonar anormal $(25,93 \% ; n=14)($ Tabela $3)$. 
Tabela 3 - Resultados descritivos da sintomatologia entre puérperas diagnosticadas com a COVID-19 durante a gravidez. Aracaju, Sergipe, Brasil, 2020.

\begin{tabular}{|c|c|c|}
\hline Sinais/Sintomas & $N=54$ & $\%$ \\
\hline \multicolumn{3}{|c|}{$\begin{array}{l}\text { Frequências individualizadas de sinais/sintomas obstétricos que } \\
\text { motivaram a consulta }\end{array}$} \\
\hline Pressão Arterial elevada & 17 & 31,48 \\
\hline Dor em Baixo Ventre & 21 & 38,89 \\
\hline Disúria & 03 & 5,56 \\
\hline Cefaleia & 20 & 37,04 \\
\hline Sangramento & 03 & 5,56 \\
\hline Epigastralgia & 05 & 9,26 \\
\hline Perda de líquido & 11 & 20,37 \\
\hline Crise convulsiva & 01 & 1,85 \\
\hline Amaurose & 01 & 1,85 \\
\hline Escotomas & 02 & 3,70 \\
\hline Anasarca & 02 & 3,70 \\
\hline \multicolumn{3}{|c|}{ Frequências individualizadas de sinais/sintomas da COVID-19 } \\
\hline Coriza & 19 & 35,19 \\
\hline Cefaleia & 28 & 51,85 \\
\hline Tosse & 24 & 44,44 \\
\hline Febre & 28 & 51,85 \\
\hline Calafrios & 06 & 11,11 \\
\hline Astenia & 06 & 11,11 \\
\hline Inapetência & 02 & 3,70 \\
\hline Dor de garganta & 09 & 16,67 \\
\hline Mialgia & 10 & 18,52 \\
\hline Diarreia & 07 & 12,96 \\
\hline Dispneia & 11 & 20,37 \\
\hline Vômito/Náusea & 09 & 16,67 \\
\hline Erupção cutânea & 02 & 3,70 \\
\hline Odinofagia & 01 & 1,85 \\
\hline Anosmia & 08 & 14,81 \\
\hline Disgeusia & 06 & 11,11 \\
\hline Artralgia & 01 & 1,85 \\
\hline \multicolumn{3}{|c|}{$\begin{array}{l}\text { Frequências individualizadas de sinais/sintomas de gravidade da } \\
\text { COVID-19 }\end{array}$} \\
\hline Saturação $\leq 94 \%$ em ar ambiente & 06 & 11,11 \\
\hline Ausculta pulmonar anormal & 14 & 25,93 \\
\hline Aumento da Frequência Cardíaca & 15 & 27,78 \\
\hline Aumento da Frequência Respiratória & 09 & 16,67 \\
\hline Oxigenoterapia & 07 & 12,96 \\
\hline Aumento de Proteína C Reativa & 30 & 55,56 \\
\hline Ventilação mecânica & 06 & 11,11 \\
\hline Internação em UTI & 07 & 12,96 \\
\hline Aumento de desidrogenase láctica (DHL) & 01 & 1,85 \\
\hline Aumento de velocidade de hemossedimentação (VHS) & 05 & 9,26 \\
\hline Coma & 01 & 1,85 \\
\hline Leucocitose & 20 & 37,04 \\
\hline Plaquetopenia & 02 & 3,70 \\
\hline
\end{tabular}

Nota: N= Frequência absoluta, \%= Frequência relativa. Fonte: Serviço de Arquivo Médico - SAME (2020).

Em relação ao perfil dos nascimentos, nota-se que 21,4\% (n=9) destes recém-nascidos (RN) obtiveram Apgar $\leq 6$ no primeiro minuto e $56,4 \%(\mathrm{n}=22)$ nasceram com idade gestacional < 37 semanas. Na avaliação do resultado do exame RT-PCR, 
ressalta-se que 42,86\% ( $\mathrm{n}=18) \mathrm{RN}$ não foram testados, porém, dentre os testados, 38,10\% (n=16) recém-nascidos apresentaram resultado positivo para COVID-19 (Tabela 4).

\begin{tabular}{|c|c|c|}
\hline Perfil do nascimento & $\mathrm{N}=42$ & $\%$ \\
\hline Apgar $1^{\circ}$ minuto & & \\
\hline $0-3$ & 04 & 9,52 \\
\hline $4-6$ & 05 & 11,90 \\
\hline $7-10$ & 32 & 76,19 \\
\hline Ignorado & 01 & 2,38 \\
\hline Apgar $5^{\circ}$ minuto & & \\
\hline $0-3$ & 00 & 0,00 \\
\hline $4-6$ & 06 & 14,28 \\
\hline $7-10$ & 35 & 83,33 \\
\hline Ignorado & 01 & 2,38 \\
\hline Classificação do recém-nascido segundo Idade Gest & & \\
\hline Pré-termo extremo (menos de 30 semanas de IG) & 01 & 2,56 \\
\hline Muito prematuro (de 30 a 33 semanas e 6 dias de IG) & 06 & 15,38 \\
\hline Pré-termo tardio (de 34 a 36 semanas e 6 dias de IG) & 15 & 38,46 \\
\hline A termo (de 37 a 41 semanas e 6 dias de IG) & 17 & 43,59 \\
\hline Pós-termo (42 semanas ou mais de IG) & 00 & 0,00 \\
\hline Resultado do exame RT-PCR no recém-nascido & & \\
\hline Positivo para COVID-19 & 16 & 38,10 \\
\hline Negativo para COVID-19 & 08 & 19,05 \\
\hline Não realizado & 18 & 42,86 \\
\hline
\end{tabular}

Nota: N= Frequência absoluta, \%= Frequência relativa. Fonte: Serviço de Arquivo Médico - SAME (2020).

\section{Discussão}

Neste estudo foram analisados os prontuários de 54 gestantes. Dentre estas, houve predomínio de grávidas concentradas na faixa etária de 20 a 34 anos, o que corrobora com dados do Sistema de Informação da Vigilância Epidemiológica da Gripe (SIVEP-GRIPE), em que a faixa etária mais acometida por COVID-19 no ano de 2020 foi a de 20 a 29 anos de idade com 2.009 (41,2\%) casos, seguida da faixa etária de 30 a 39 anos, com 1.962 (40,2\%) casos (Brasil, 2021).

A maioria das gestantes se autodeclararam da raça parda, seguida da preta, em consonância, também, com os dados do SIVEP-GRIPE, no qual a cor da pele mais frequente foi à parda (Brasil, 2021); e com a pesquisa de Knight et al. (2020), a qual mostrou que 56\% das grávidas admitidas com SARS-CoV-2 eram pretas ou pertenciam a minorias étnicas. Esse fato representa um fator de risco para adquirir o coronavírus, pois, historicamente, mulheres pardas e pretas sofrem com as iniquidades para acessar o sistema de saúde, visto que gestantes negras têm maior dificuldade de acesso ao pré-natal e representam cerca de $65 \%$ das mortes maternas no país (Lima, 2021).

Quanto ao trimestre gestacional em que as participantes estavam ao contraírem COVID-19, houve um predomínio de grávidas no último trimestre. Este dado coincide com o Sistema de Informação do Ministério da Saúde que analisa todas as gestantes positivas, através da ficha de notificação para SRAG, no qual houve 3.028 (62,0\%) casos com idade gestacional no $3^{\circ}$ trimestre (Brasil, 2021). 
A gestação por si só, torna a mulher mais suscetível a agravos de saúde, e mesmo os sintomas leves de COVID-19 podem trazer consequências importantes para o desfecho obstétrico (Panahi et al., 2020). A infecção pelo Sars-Cov2 está associada à morte fetal, ao maior risco de desenvolver parto prematuro, pré-eclâmpsia, eclâmpsia, síndrome HELLP, infarto do miocárdio e tromboembolismo venoso e necessidade de uma cesariana de emergência, em comparação com mulheres grávidas sem infecção por COVID-19 (Knight et al., 2020. Ko et al., 2021. Elshafeey et al., 2020 \& Jering et al., 2021).

Ademais, durante a gestação, ocorrem alterações anatômicas e fisiológicas da gravidez em diversos sistemas como o cardiovascular, respiratório, imunológico e de coagulação. Essas modificações tornam a gestante mais suscetível a pneumonias virais e, consequentemente, aumentam o risco de complicações e óbitos em gestantes que contraíram o coronavírus. A fisiologia pulmonar durante a gravidez sofre alterações hormonais e funcionais que tornam as gestantes menos tolerantes à hipóxia. Desde o início da gravidez, os níveis de progesterona atuam no tronco encefálico aumentando a frequência respiratória e o volume corrente, a complacência da parede torácica diminui e a resistência das vias aéreas também (Czeresnia et al., 2020).

No último trimestre, o útero restringe o diafragma, o que diminui a capacidade pulmonar total. Essas adaptações respiratórias associadas às alterações imunológicas colocam as gestantes em risco de desenvolver infecções respiratórias mais graves, podendo acarretar uma hipoxemia, que surge de uma infecção pulmonar, podendo levar à vasoconstrição e RCIU (Czeresnia et al., 2020).

Essa suscetibilidade ocorre, principalmente, quando a gestante está no último trimestre de gestação ou no período do puerpério porque as alterações anatômicas e fisiológicas que ocorrem no organismo materno atingem o seu pico durante esse período (Allotey et al., 2020). Esse achado corrobora com a realidade local, pois a maior parte das pacientes apresentaram pelo menos algum sinal de gravidade, como aumento da proteína $\mathrm{C}$ reativa (PCR) e leucocitose, em exame laboratorial; elevação da frequência cardíaca e alteração da ausculta respiratória. Apenas 7 (12,9\%) pacientes não apresentaram sintomas de gravidade.

Em relação aos sinais e sintomas das gestantes hospitalizadas observou-se que o sintoma mais frequente foi a cefaleia, seguido por febre, tosse, coriza, dispneia e mialgia, dados que estão em conformidade com o Ministério da Saúde, no qual a sintomatologia mais frequente apresentada pelas grávidas foram tosse, febre, dispneia e desconforto respiratório. Nos casos que evoluíram ao óbito, além desses sintomas, 66\% apresentaram saturação de oxigênio < 95\% (Brasil, 2021).

Ademais, em uma revisão robusta publicada em setembro de 2020, uma coorte de 77 estudos perfazendo um total de 13.118 mulheres apresentam conclusões semelhantes quanto à sintomatologia e o acometimento de mulheres grávidas com COVID-19: a febre foi a manifestação mais frquente (40\%) e a tosse foi a segunda manifestação clínica mais frequente (39\%) (Allotey et al., 2020). Em duas revisões sistemáticas que envolveram 266 e 441 pacientes, respectivamente, também demonstraram que os sintomas mais frequentes foram tosse, febre, dispneia e mialgia (Zamaniyan et al., 2020; Juan et al., 2020).

Uma revisão sistemática de Zaigham e colaboradores incluiu 108 casos de COVID-19 na gravidez tendo como principais sintomas a febre (68\%), tosse (34\%), mal-estar (13\%), dispneia (12\%) e diarreia (6\%), sintomatologia que se assemelha aos sintomas em pacientes não grávidas (Zaigham \& Andersson, 2020).

A febre e a hipoxemia são responsáveis pelo aumento do risco de trabalho de parto prematuro, rotura prematura de membrana, prematuridade, cesariana (Czeresnia et al, 2020) e aumento do risco de complicações fetais. Allotey et al. (2020) demonstrou, através do seu estudo, que o trabalho de parto prematuro foi a complicação mais prevalente entre as pacientes que desenvolveram COVID-19 e, consequentemente a necessidade de cuidados intensivos neonatais foi mais frequente nos recémnascidos dessas mães acometidas por COVID-19 e os índices de cesarianas nessas pacientes foram mais elevados.

Neste estudo, a maioria dos partos foram realizados via cirúrgica. Esse dado leva ao questionamento da indicação da via de parto, visto que, os altos índices de cesárea também apareceram em diversas pesquisas realizadas, como na de Elshafeey 
e colaboradores (2020) que conduziram uma revisão sistemática com 385 casos de COVID-19 na gravidez. Nesta revisão, foram relatados 252 partos, 175 cesáreas $(69,4 \%)$ e 77 vaginais $(30,6 \%)$.

O momento e a via de parto, na maioria dos casos, não devem ser determinados pela infecção materna por SARSCoV-19. Entretanto, indica-se antecipação do parto e a realização de cesárea no caso de gestantes que evoluam com sintomas graves ou críticos com agravamento da situação clínica, ou nos casos de sofrimento fetal. A via de parto deve ser baseada em indicação obstétrica, seja materna ou fetal, e para definir é necessária uma avaliação multidisciplinar, considerando estado geral da paciente, idade gestacional e vitalidade fetal. Em gestantes com boas condições clínicas, sintomas leves e feto com boa vitalidade, o parto vaginal é seguro e recomendável (Gajbhiye et al., 2020. The American College of Obstetricians and Gynecologists, 2020).

Os indivíduos acometidos pelo coronavírus apresentam alterações nos níveis de albumina, elevação de PCR, Ddímero, DHL, VHS e uma linfopenia. Cerca de $22 \%$ dos pacientes apresentam elevação das enzimas hepáticas e habitualmente as plaquetas estão no limite inferior da normalidade (Huang et al., 2020).

Essa sintomatologia pode ser explicada pelo mecanismo de ação do vírus SARS-CoV-2, visto que ele invade o hospedeiro através do receptor de entrada na célula da enzima conversora da angiotensina 2, levando à disfunção do sistema renina angiotensina aldosterona e, então, à vasoconstrição. Em contraste, os sinais e sintomas compatíveis com o diagnóstico de Pré-Eclâmpsia e Síndrome HELLP podem ser derivados da disfunção renal e cardiovascular para infecção grave do SARSCoV-2 (Mendoza et al., 2020).

Dessa maneira, a PE é, em suma, um resultado do dano endotelial originado pelo estresse oxidativo da placenta e estado antiangiogênico, que leva ao aparecimento de hipertensão e proteinúria, enzimas hepáticas elevadas, insuficiência renal, trombocitopenia, entre outros. Logo, os achados clínicos de COVID-19 e PE podem ser muito semelhantes, sendo desafiador diferenciá-las (Mendoza et al., 2020).

Desse modo, os profissionais de saúde devem estar cientes da existência da síndrome semelhante à PE induzida por COVID-19 e monitorar as gestações com suspeita de pré-eclâmpsia de forma cautelosa. Ao contrário da PE grave, a síndrome pelo coronavírus pode não ser uma indicação para antecipação de parto, pois não havendo complicação placentária, pode-se resolver espontaneamente após a recuperação da pneumonia grave (Mendoza et al., 2020).

Apesar da COVID-19 cursar com sintomas leves na maioria das gestantes infectadas, quando comparadas às mulheres de mesma idade não gestantes, as parturientes apresentam maiores taxas de internação e necessidade de ventilação mecânica (Jering et al., 2021).

Neste estudo podemos observar que uma parcela das gestantes foram internadas em UTI e apresentaram saturação menor que $95 \%$ em ar ambiente e, consequentemente, utilizaram ventilação mecânica. Semelhante aos resultados encontrados na revisão sistemática conduzida por Elshafeey e colaboradores (2020), que dentre 385 casos de COVID-19, 368 (95,6\%) casos foram classificados como leves, 14 (3,6\%) como graves e 3 (0,8\%) como críticos; 17 (4,4\%) necessitaram de UTI e todos, exceto 1, recuperados.

Com relação ao desfecho das gestantes, entre as pacientes participantes do estudo de Jering e colaboradores (2021), a taxa de mulheres com COVID-19 que necessitou de cuidados intensivos foi de 3,3\%, sendo apenas 1,3\% de ventilação mecânica, que apesar de parecer pouco mostrou um OR (OddsRatio) 23,7 ao comparar com mulheres não gestantes. Nove pacientes com COVID-19 (0,1\%) morreram na internação, analisando este dado em mortes por 100.000, a diferença é de 141 mortes entre as infectadas para 5 mortes entre as não portadoras de COVID-19. Dessa maneira, pode-se perceber que há um risco maior para ventilação mecânica e mortalidade materna em grávidas portadoras de COVID-19.

As comorbidades, como diabetes, hipertensão e obesidade, são fatores de risco para resultados adversos em pacientes afetados por coronavírus (Elshafeey et al., 2020. Jering et al., 2021). Dentre as participantes analisadas neste estudo realizado 
em Sergipe, verificou-se que grande parte das gestantes possuíam comorbidades. Entre estas, as mais prevalentes foram: Hipertensão Crônica, Diabetes Mellitus Gestacional, Hipertensão Gestacional e Asma.

Na revisão publicada por Allotey e colaboradores (2020), analisou 77 estudos perfazendo um total de 13.118 mulheres e chegaram à conclusão que a idade materna avançada, índice de massa corpórea elevada e comorbidades prévias, sendo diabetes prévio a mais prevalente, foram os fatores de risco mais relevantes nas pacientes gestantes que desenvolveram doença mais grave necessitando de cuidados intensivos, indicando possivelmente um perfil pré-definido que contribuem para um maior risco de resultados maternos graves, como admissão em UTI e deterioração clínica que pode levar ao óbito.

Diabetes mellitus, obesidade, hipertensão, imunossupressão foram preditores independentes significativos para intubação, enquanto a gravidez, diabetes mellitus, doenças cardiovasculares, obesidade foram preditores independentes significativos para admissão na UTI (Verity et al., 2020. Martinez-Portilla et al., 2021).

Com relação aos fatores sociodemográficos, as gestantes brasileiras que evoluíram para óbito por SRAG confirmado para COVID-19 (1.041 óbitos), tiveram como faixa etária mais acometida a de 30 a 39 anos com 541 (52,0\%) óbitos, também seguida pela faixa etária de 20 a 29 anos, com 326 (31,3\%) óbitos; a cor mais frequente é a parda (45,8\%; n=477), e mais da metade das gestantes com SRAG por Covid-19 estavam no $3^{\circ}$ trimestre de gestação (55,2\%; n=575) (Brasil, 2021).

No que tange a mortalidade materna, ocorreram 3 óbitos maternos durante a coleta desta pesquisa. Todas gestantes de $3^{\circ}$ trimestre, na faixa etária de 24-39 anos, cor da pele predominantemente parda, submetidas à cesárea, portadoras de comorbidades (HAC, Cardiopatia e Diabetes Mellitus Gestacional), com hipóteses diagnósticas relacionadas às Síndromes Hipertensivas (Pré-Eclâmpsia Leve, Síndrome HELLP e Eclâmpsia), Cardiopatia e Diabetes Mellitus Gestacional. Também foram pacientes que necessitaram de ventilação mecânica e internação em UTI, e uma foi a óbito no próprio centro cirúrgico.

É importante ressaltar que, apesar da maternidade onde foi realizado o estudo ser de alto risco obstétrico, não há UTI materna. Quando é necessário realizar o procedimento de intubação, este é feito na sala de cirurgia e as pacientes ficam aguardando vaga através do sistema de regulação de leitos de UTI para serem transferidas. Esses dados sugerem que pacientes obstétricos podem enfrentar atrasos e barreiras para acessar ventiladores mecânicos e terapia intensiva.

As limitações deste estudo estão relacionadas à obtenção dos dados a partir da análise de prontuários e da ficha de notificação para SRAG, sem a obtenção do relato das gestantes/puérperas. Esse fato resulta em muitas informações ignoradas porque não foram preenchidas no ato da notificação e por também não constar em prontuário. Ademais, houve uma dificuldade para localizar e acessar os prontuários, visto que a maternidade onde foi realizada a coleta não possui prontuário eletrônico.

No presente estudo, verificou-se que as frequências das características clínicas e epidemiológicas, fatores de risco e desfecho da COVID-19 nas gestantes hospitalizadas foram compatíveis com aquelas encontradas na literatura. Ou seja, são pacientes entre 20 a 34 anos, com cor da pele parda, presença de comorbidades prévias e que apresentaram sintomas de gravidade para COVID-19. No entanto, a maioria das gestantes evoluíram bem e tiveram como desfecho a alta, apenas 7 precisaram ser admitidas em UTI.

Sugere-se ainda, por fim, a realização de estudos com desenhos metodológicos e análises mais robustas que possam mensurar as questões que precisam ser respondidas sobre a gravidez nesta pandemia, incluindo transmissão vertical, dano placentário e receptores, testes de diagnóstico para doença grave, efeitos de longo prazo e, especialmente, vacinação na gravidez, para um melhor entendimento dos desfechos clínicos maternos, fetais e neonatais da SARS-CoV-2, a fim de contribuir para tomada de decisões terapêuticas e de precaução da infecção.

\section{Conclusão}

Conclui-se que políticas de saúde devem ser direcionadas às gestantes com maior risco de adoecimento, levando-se em consideração aspectos sociais, econômicos e demográficos. A abordagem dos problemas sociodemográficos pelas 
autoridades deve ser preventiva e constante, no sentido de que se apresentam como fatores de risco para diversos tipos de adoecimento, incluindo a COVID-19.

Portanto, a assistência pré-natal tem importância fundamental para prevenção da morbimortalidade materna, principalmente quando o acompanhamento é iniciado em tempo oportuno, ou seja, no primeiro trimestre da gravidez, pois objetiva prevenir complicações e tratar as patologias em tempo hábil (Brasil, 2021).

\section{Referências}

Allotey, J, Stallings, E., Bonet, M., Yap, M., Chatterjee, S., Kew, T. \& Thangaratinam, S. (2020). Clinical manifestations, risk factors, and maternal and perinatal outcomes of coronavirus disease 2019 in pregnancy: living systematic review and meta-analysis. The BMJ, 370, m3320. https://www.bmj.com/content/370/bmj.m3320.

Amorim, M. M. R., Melo, A. S. O., Neto, A. H. F., \& Oliveira, T. V. (2020). Protocolo de atendimento de pacientes com covid-19 (infecção suspeita ou confirmada) do instituto de saúde Elpídio de Almeida - ISEA. http://rehuna.org.br/wp-content/uploads/2020/04/PROTOCOLO-DE-CRISE-COVID19ISEA.pdf

Brasil. Conselho Nacional de Saúde. (2012). Resolução no 466, de 12 de dezembro de 2012. https://wp-sites.info.ufrn.br/admin/facisa/wpcontent/uploads/sites/4/2020/07/RESOLU\%C3\%87\%C3\%95ES-466-12-510-16-e-580-18.pdf.

Brasil. Ministério da Saúde. Secretaria de Vigilância em Saúde. (2021). Boletim Epidemiológico Especial COE- COVID-19 no 44. https://www.gov.br/saude/pt-br/media/pdf/2021/janeiro/07/boletim_epidemiologico_covid_44.pdf

Brasil. Ministério da Saúde. Secretaria de Vigilância em Saúde. (2020). Boletim Epidemiológico Especial COE- COVID-19 nº 21. http://antigo.saude.gov.br/images/pdf/2020/July/08/Boletim-epidemiologico-COVID-21-corrigido-13h35--002-.pdf

Brasil. Ministério da Saúde. Secretaria de Vigilância em Saúde. (2021). Boletim Epidemiológico Especial COE- COVID-19 nº 86. https://www.gov.br/saude/pt-br/media/pdf/2021/outubro/29/boletim_epidemiologico_covid_86-final-_29out.pdf

Brasil. Ministério da Saúde. Secretaria de Atenção Primária à Saúde. (2021). Manual de recomendações para a assistência à gestante e puérpera frente à pandemia de Covid-19. (2a ed.), Ministério da Saúde, 2021. https://portaldeboaspraticas.iff.fiocruz.br/wpcontent/uploads/2021/09/manual_assistencia_gestante.pdf.

Czeresnia, R. M., Trad, A. T. A., Britto, I. S. W., Negrini, R., Nomura, M. L., Pires, P. \& Ruano R. (2020). SARS-CoV-2 and Pregnancy: A Review of the Facts. Revista Brasileira de Ginecologia e Obstetrícia, 42 (9), 562-568. http://www.scielo.br/scielo.php?script=sci_arttext\&pid=S0100$72032020000900562 \& \operatorname{lng}=$ en.

Dashraath, P., Wong, J. L. J., Lim, M. X. K., Lim, L. M., Li, S., Biswas, A. \& Su, L. L. (2020). Coronavirus disease 2019 (COVID-19) pandemic and pregnancy. American Journal of Obstetrics \& Gynecology, 222(6), 521-531. https://www.ajog.org/article/S0002-9378(20)30343-4/fulltext.

Elshafeey, F., Magdi, R., Hindi, N., Elshebiny, M., Farrag, N., Mahdy, S. \& Nabhan, A. (2020). A systematic scoping review of COVID-19 during pregnancy and childbirth. International Journal of Gynecology \& Obstetrics, 150(01), 47-52. https://obgyn.onlinelibrary.wiley.com/doi/abs/10.1002/ijgo.13182.

Fiocruz. (2020). Pesquisa Nascer no Brasil: Inquérito Nacional sobre Parto e Nascimento. http://www6.ensp.fiocruz.br/nascerbrasil/questionarios-eletronicos/

Gajbhiye, R. K., Modi, D. N., \& Mahale, S. D. (2020). Pregnancy outcomes, Newborn complications and Maternal-Fetal Transmission of SARS-CoV-2 in women with COVID-19: a systematic review of 441 cases. The BMJ. https://www.medrxiv.org/content/10.1101/2020.04.11.20062356v2.

Huang, C., Wang, Y., Li, X., Ren, L., Zhao J., Hu, Y., \& Cao B. (2020). Clinical features of patients infected with 2019 novel coronavirus in Wuhan, China. The Lancet, 395, 497-506. https://www.thelancet.com/journals/lancet/article/PIIS0140-6736(20)30183-5/fulltext.

Jering, K. S., Claggett, B. L., Cunningham, J. W., Rosenthal, N., Vardeny, O., Greene, M. F., \& Solomon, S. D. (2021). Clinical Characteristics and Outcomes of Hospitalized Women Giving Birth With and Without Covid-19. JAMA Internal Medicine, 181 (5), 714-717. https://jamanetwork.com/journals/jamainternalmedicine/fullarticle/2775396.

Juan, J., Gil, M. M., Rong, Z., Zhang, Y., Yang H., \& Poon L. C. (2020). Effects of Coronavirus Disease 2019 (COVID-19) on Maternal, Perinatal and Neonatal Outcomes: a Systematic Review of 266 Pregnancies. The BMJ, 56,15-27. https://www.medrxiv.org/content/10.1101/2020.05.02.20088484v1.

Knight, M., Bunch, K., Vousden, N., Morris, E., Simpson, N., Gale, C. \& Kurinczuk, J. J. (2020). Characteristics and outcomes of pregnant women admitted to hospital with confirmed SARS-CoV-2 infection in UK: national population based cohort study. The BMJ, 369 , m2107. https://www.bmj.com/content/369/bmj.m2107.

Ko, J.Y., DeSisto, C. L., Simeone, R. M., Ellington, S., Galang, R. R., Oduyebo, T., \& Shapiro-Mendoza, C. K. (2021). Adverse pregnancy outcomes, maternal complications, and severe illness among US delivery hospitalizations with and without a Coronavirus disease 2019 (COVID-19) diagnosis. Clinical Infectious Diseases, 73, S24-31. https://academic.oup.com/cid/article/73/Supplement_1/S24/6274300.

Lima, R. C. H. M. (2021). Cartilha Direito Sexuais e Reprodutivos das Mulheres. Brasília: Escola de Assistência Jurídica da Defensoria Pública. https://www.planaltina.df.gov.br/wp-conteudo//uploads/2021/07/Cartilha_Direito_Sexuais_e_Reprodutivos_das_Mulheres.pdf.

Martinez-Portilla, R. J., Sotiriadis, A., Chatzakis, C., Torres-Torres, J., Espino Y Sosa, S., Sandoval-Mandujano, K., \& Poon, L. C. (2021). Pregnant women with SARS-CoV-2 infection are at higher risk of death and pneumonia: propensity score matched analysis of a nationwide prospective cohort (COV19Mx). Ultrasound in Obstetrics \& Ginecology, 57(2), 224-231. https://pubmed.ncbi.nlm.nih.gov/33320401/. 
Mendoza, M., Garcia-Ruiz, I., Maiz, N., Rodo, C., Garcia-Manau, P., Serrano, B., \& Suy, A. (2020). Pre-eclampsia-like syndrome induced by severe COVID19: a prospective observational study. An International Journal of Obstetrics and Gynaecology, 127(11), 1374-1380. https://obgyn.onlinelibrary.wiley.com/doi/10.1111/1471-0528.16339.

Organização Mundial da Saúde. (2020). Q\&A on coronaviruses (COVID-19). Genebra. https://www.who.int/emergencies/diseases/novel-coronavirus2019/question-and-answers-hub/q-a-detail/q-a-coronaviruses.

Panahi, L., Amiri, M., \& Pouy, S. (2020). Risks of Novel Coronavirus Disease (COVID-19) in Pregnancy: a Narrative Review. Archives of Academic Emergency Medicine, 8(1), e34. https://www.ncbi.nlm.nih.gov/pmc/articles/PMC7092922/.

Schwartz, D. A., \& Dhaliwal, A. (2020). Infections in pregnancy with covid-19 and other respiratory rna virus diseases are rarely, if ever, transmitted to the fetus: experiences with coronaviruses, HPIV, hMPV RSV, and Influenza. Archives of Pathology \& Laboratory Medicine, 144, 920-8. https://meridian.allenpress.com/aplm/article/144/8/920/442144/Infections-in-Pregnancy-With-COVID-19-and-Other.

Souza, A. S. R., \& Amorim, M. M. R. (2021). Mortalidade materna pela COVID-19 no Brasil. Revista Brasileira de Saúde Materno Infantil, 21(Suppl1), 253256. http://www.scielo.br/scielo.php?script=sci_arttext\&pid=S1519-38292021000100253\&lng=pt.

Takemoto, M. L. S., Menezes, M. O., Andreucci, C. B., Nakamura-Pereira, M., Amorim, M. M., Katz, L., \& Knobel, R. (2020). The tragedy of COVID-19 in Brazil: 124 maternal deaths and counting. International Journal of Gynecology \& Obstetrics, 151, 154-6. https://obgyn.onlinelibrary.wiley.com/doi/full/10.1002/ijgo.13300.

The American College of Obstetricians and Gynecologists (ACOG). (2020). COVID-19 FAQs for Obstetrician-Gynecologists, Obstetrics. /www.acog.org/clinical-information/physician-faqs/covid-19-faqs-for-ob-gyns-obstetrics.

Verity, R., Okell, L. C., Dorigatti, I., Winskill, P., Whittaker, C., Imai, N., \& Ferguson, N. M. (2020). Estimates of the severity of coronavirus disease 2019: a model-based analysis. The Lancet Infections Diseases, 20, 669-677. https://www.thelancet.com/journals/laninf/article/PIIS1473-3099(20)30243-7/fulltext.

Wen, R., Sun, P., \& Xing, Q-S. (2020). A Patient with SARS-CoV-2 Infection during Pregnancy in Qingdao, China. Journal of Microbiology, Immunology and Infection, 3-4. https://doi.org/10.1016/j.jmii.2020.03.004.

Yu, N., Li, W., Kang, Q., Xiong, Z., Wang, S., Lin, X., \& Wu, J. (2020). Clinical features and obstetric and neonatal outcomes of pregnant patients with COVID-19 in Wuhan, China: a retrospective, single-centre, descriptive study. The Lancet Infections Diseases, 3099(20), 1-6. https://www.thelancet.com/journals/laninf/article/PIIS1473-3099(20)30176-6/fulltext.

Zaigham, M., \& Andersson, O. (2020). Maternal and perinatal outcomes with COVID-19: A systematic review of 108 pregnancies. Acta Obstetricia ET Gynecologica Scandinavica, 99(07), 823-829. https://obgyn.onlinelibrary.wiley.com/doi/10.1111/aogs.13867.

Zamaniyan, M., Ebadi, A., Aghajanpoor, M. S., Rahmani, Z., Haghshenas, M., \& Azizi, S. (2020). Preterm delivery in pregnant woman with critical COVID19 pneumonia and vertical transmission. Prenatal Diagnosis, 40, 1759-1761. https://obgyn.onlinelibrary.wiley.com/doi/full/10.1002/pd.5713. 